\title{
Electrothermal stress in conductive body with collinear cracks
}

\author{
P. Wang ${ }^{\mathrm{a}, \mathrm{b}, *}$, Z.G. Tian ${ }^{\mathrm{a}, \mathrm{b}}$, X.Z. Bai ${ }^{\mathrm{a}, \mathrm{b}}$

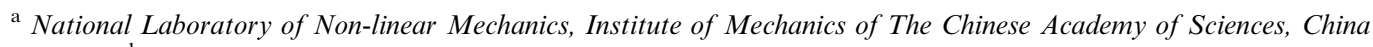 \\ b Yanshan University, College of Civil Engineering and Mechanics, Qinhuangdao 066004, China
}

\begin{abstract}
The temperature and stress field in a thin plate with collinear cracks interrupting an electric current field are determined. This is accomplished by using a complex function method that allows a direct means of finding the distribution of the electric current, the temperature and stress field. Temperature dependency for the heat-transfer coefficient, coefficient of linear expansion and the elastic modulus are considered. As an example, temperature distribution is calculated for an alloy (No. GH2132) plate with two collinear cracks under high temperature. Relationships between the stress, temperature, electric density and crack length are obtained. Crack trajectories emanating from existing crack are predicted by application of the strain energy density criterion which can also be used for finding the load carrying capacity of the cracked plate.
\end{abstract}

(C) 2003 Elsevier Ltd. All rights reserved.

Keywords: Stress field; Temperature field; Electric current density; Multiple cracks; Thin conductive plate; Strain energy density

\section{Introduction}

Electromagnetic effects have numerous useful engineering applications because they can alter the strain, stress, and temperature field in the material. Magneto-thermo-elastic theory can be applied for a quantitative assessment of these effects. One such application is to arrest crack motion by changing the material properties in the immediate vicinity of the crack tips. This involves heating the local

\footnotetext{
${ }^{*}$ Corresponding author. Address: Yanshan University, College of Civil Engineering and Mechanics, Qinhuangdao 066004, China. Tel.: +86-335-8057101; fax: +86-335-8063941.

E-mail address: wangpin0721@sina.com (P. Wang).
}

material electromagnetically in a very short period of time such that sufficiently high energy can be localized to cause a change in the material microstructure. Such a change is sufficient to prevent a crack from initiating or to arrest a moving crack. For those structural components that conduct electric current, the method can be easily applied using a voltage source once cracks are detected by non-destructive testing techniques such as ultrasonic and acoustic emissions.

Addressed in this work are cracks or line defects in metals that are aligned collinearly. More specifically, consider a thin plate conducting an electric current field that is interrupted by $N$ collinear cracks. The temperature and stress field are determined for the situation when crack propagation 
is stopped by changing the material properties ahead of the crack. This is done by sending an electric current down the plate. The timing and strength of the current depend on the size of the specimen and the mechanical and thermal properties of the material. To this end, it is pertinent to have knowledge of the prevailing stress, strain and thermal field around the cracks. To demonstrate the feasibility of the proposed scheme, a high temperature resistance alloy GH2132 with two collinear cracks has been selected for this study.

\section{Temperature field with $N$ collinear cracks}

\subsection{Electric current density distribution}

Fig. 1 shows $N$ collinear cracks in a complex plane. The length of each crack is $L_{k}$. Now, let $L=\sum_{k=1}^{n} L_{k}$. When the electric current is switched on normal to the crack, the relationship between the current intensity $I$ and the current density $J$ is given by [1]

$-J_{x}=\frac{\partial I}{\partial x}, \quad J_{y}=\frac{\partial I}{\partial y}$

With reference to the complex plane $z=x+\mathrm{i} y$, an analytic function $\varphi^{\mathrm{T}}(z)=I+\mathrm{i} V$ can be defined such that $I$ and $V$ satisfy the Cauchy-Riemann condition [2]. This implies that

$-J_{x}+\mathrm{i} J_{y}=\Phi^{\mathrm{T}}(z), \quad I=\operatorname{Re}\left[\varphi^{\mathrm{T}}(z)\right]$

Here,

$\varphi^{\mathrm{T}}(z)=\int \Phi^{\mathrm{T}}(z) \mathrm{d} z, \quad \Phi^{\mathrm{T}}(z)=\frac{\partial I}{\partial x}-\mathrm{i} \frac{\partial I}{\partial y}$
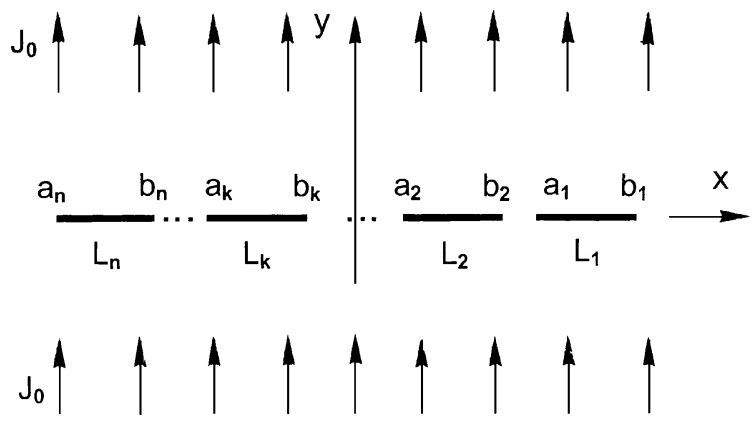

Fig. 1. A thin current carrying plate with multiple cracks.
The symbol $\mathrm{Re}[]$ stands for the real part of the function. It follows that

$|J|=\left|\sqrt{J_{x}^{2}+J_{y}^{2}}\right|=\left|\Phi^{\mathrm{T}}(z)\right|$

The boundary conditions of $L$ are given by

$$
\begin{aligned}
& {\left[\Phi^{\mathrm{T}}(s)-\bar{\Phi}^{\mathrm{T}}(s)\right]^{+}+\left[\Phi^{\mathrm{T}}(s)-\bar{\Phi}^{\mathrm{T}}(s)\right]^{-}=2 f(s)} \\
& {\left[\Phi^{\mathrm{T}}(s)+\bar{\Phi}^{\mathrm{T}}(s)\right]^{+}-\left[\Phi^{\mathrm{T}}(s)+\bar{\Phi}^{\mathrm{T}}(s)\right]^{-}=2 g(s)}
\end{aligned}
$$

where

$$
\begin{aligned}
& f(s)=\frac{1}{\mathrm{i}}\left[\left(\frac{\partial I}{\partial y}\right)^{+}+\left(\frac{\partial I}{\partial y}\right)^{-}\right] \\
& g(s)=\frac{1}{\mathrm{i}}\left[\left(\frac{\partial I}{\partial y}\right)^{+}-\left(\frac{\partial I}{\partial y}\right)^{-}\right]
\end{aligned}
$$

The solution to the Hilbert problem as given by Eq. (5) is well known:

$$
\begin{aligned}
& \Phi^{\mathrm{T}}(z)=\Phi_{0}^{\mathrm{T}}(z)+\frac{P_{n}^{\mathrm{T}}(z)}{\chi(z)} \\
& \Phi_{0}^{\mathrm{T}}(z)=\frac{1}{2 \pi \mathrm{i} \chi(z)} \int_{L} \frac{\chi^{+}(s) f(s)}{s-z} \mathrm{~d} s+\frac{1}{2 \pi \mathrm{i}} \int_{L} \frac{g(s)}{s-z} \mathrm{~d} s
\end{aligned}
$$

Note that the branch cuts for the cracks are given by

$$
\begin{aligned}
& \chi(z)=\prod_{j=1}^{n}\left(z-a_{j}\right)^{1 / 2}\left(z-b_{j}\right)^{1 / 2} \\
& P_{n}^{\mathrm{T}}(z)=c_{0} z^{n}+c_{1} z^{n-1}+\cdots+c_{n}
\end{aligned}
$$

The current density is given by

$c_{0}=\frac{1}{\mathrm{i}}\left(\frac{\partial I}{\partial y}\right)^{\infty}=-i J_{0}$

Similarly, the coefficients $c_{1}, c_{2}, \ldots, c_{n}$ can be determined by using the current intensity condition:

$\operatorname{Re}\left[\oint \Phi^{\mathrm{T}}(z) \mathrm{d} z\right]=0$

while using Eqs. (6) and (5). It follows that

$f(s)=0, \quad g(s)=0$

This gives a system of $N$ linear equations solving for the $N$ unknowns $c_{1}, c_{2}, \ldots, c_{n}$. The current intensity modulus can then be found by using Eq. (4). 


\subsection{Heat source}

From the principle of electromagnetic heating, it is known that heat can be produced. when current flows in a conductor. The greater the current intensity, the higher is the temperature. The current intensity increases rapidly near the crack tips with the crack length being a factor. Hence, the temperature near the tip is intensified locally. The temperature field caused by the current is therefore assumed to be controlled by the point-heating source at the crack tip. The thin plate should have a high electrical conductivity while the remaining electromagnetic heating effects can be ignored. The power of each heating source at a crack tip is given by [5]

$$
\begin{aligned}
& Q\left(a_{k}\right)=\frac{1}{\sigma} \int_{-h}^{h}\left|J\left(a_{k}\right)\right|^{2} \mathrm{~d} z=\frac{1}{\sigma} \int_{-h}^{h}\left|\Phi^{\mathrm{T}}\left(a_{k}\right)\right|^{2} \mathrm{~d} z \\
& Q\left(b_{k}\right)=\frac{1}{\sigma} \int_{-h}^{h}\left|J\left(b_{k}\right)\right|^{2} \mathrm{~d} z=\frac{1}{\sigma} \int_{-h}^{h}\left|\Phi^{\mathrm{T}}\left(b_{k}\right)\right|^{2} \mathrm{~d} z
\end{aligned}
$$

where $h$ is half of the thickness of the plate and $\sigma$ is the electrical conductivity.

\subsection{The temperature field}

When the current flows in the thin plate with $N$ collinear cracks, the resulting influence on the temperature field is the same as that of a heat source with a particular power setting. So the problem is to calculate the temperature field resulting from $2 n$ heat sources. It has two parts:

$T(z)=T_{0}(z)+T_{*}(z)$

Here, $T_{0}(z)$ is the temperature in the absence of the cracks and $T_{*}(z)$ is the correction for the presence of the cracks.

Solution of $T_{0}(z)$. The temperature $T_{0}(z)$ can be easily found and it is given by the complex functions [6]:

$$
\begin{aligned}
& T_{0}(z)=2 \operatorname{Re}\left[F_{0}(z)\right], \\
& F_{0}(z)=\sum_{k=1}^{n}\left[m_{a_{k}} \ln \left(z-a_{k}\right)+m_{b_{k}} \ln \left(z-b_{k}\right)\right]
\end{aligned}
$$

where

$$
m_{a_{k}}=-\frac{Q\left(a_{k}\right)}{4 \pi \lambda}, \quad m_{b_{k}}=-\frac{Q\left(b_{k}\right)}{4 \pi \lambda}
$$

Note that $\lambda$ is the thermal heat conduction coefficient. $Q\left(a_{k}\right)$ and $Q\left(b_{k}\right)$ are the power of the heat source at the right or left tip of a crack. They can be calculated from Eq. (12) as

$$
\begin{aligned}
T_{0}(x, y)= & \sum_{k=1}^{n}\left\{m_{a_{k}} \ln \left[\left(x-a_{k}\right)^{2}+y^{2}\right]\right. \\
& \left.+m_{b_{k}} \ln \left[\left(x-b_{k}\right)^{2}+y^{2}\right]\right\} \\
\frac{\partial T_{0}(z)}{\partial n}= & \frac{\partial T_{0}(z)}{\partial y}=\sum_{k=1}^{n} 2 y\left[\frac{m_{a_{k}}}{\left(z-a_{k}\right)^{2}-y^{2}}+\frac{m_{b_{k}}}{\left(z-b_{k}\right)^{2}-y^{2}}\right]
\end{aligned}
$$

Solution of $T_{*}(z)$. Assume the cracks are insulated such that heat cannot flow across the crack surfaces. The heat boundary conditions are

$\lambda\left(\frac{\partial T(\tau)}{\partial n}\right)=0, \quad \tau \in L$

From Eqs. (13) and (18), the following equation can be obtained:

$$
\lambda\left(\frac{\partial T_{*}(\tau)}{\partial n}\right)=-\lambda\left(\frac{\partial T_{0}(\tau)}{\partial n}\right), \quad \tau \in L
$$

Hence $T_{*}(z)$ is obtained [5]:

$$
\begin{aligned}
& T_{*}(z)=2 \operatorname{Re}\left[F_{*}(z)\right] \\
& F_{*}(z)=\frac{c}{2}+\frac{1}{2 \pi \mathrm{i}} \int_{L} \frac{\varphi(\tau)}{\tau-z} \mathrm{~d} \tau, \quad \tau \in L
\end{aligned}
$$

where $c$ is a real content. It is twice the temperature of the plate without cracks and without heat sources. $\varphi(\tau)$ is a real function defined on the boundary $L$. On $L$, it is found that

$$
\begin{aligned}
\mp \varphi\left(\tau_{0}\right) & =-2|y| \sum_{k=1}^{n}\left[\frac{m_{a_{k}}}{\left(z-a_{k}\right)^{2}-y^{2}}+\frac{A_{b_{k}}}{\left(z-b_{k}\right)^{2}-y^{2}}\right] \\
& =-2 b \sum_{k=1}^{n}\left[\frac{A_{a_{k}}}{\left(z-a_{k}\right)^{2}-y^{2}}+\frac{A_{b_{k}}}{\left(z-b_{k}\right)^{2}-y^{2}}\right]
\end{aligned}
$$

with $b$ being the width of the cracks.

Consider the initial temperature of the plate as 0 ${ }^{\circ} \mathrm{C}$, so that $c=0$. It is also known that $b=0$. According to Eq. (19), it is known that $T_{*}(z)=0$. This means: when a point-heating source is collinear with a crack, the crack has no influence on the temperature field. From Eq. (12) the temperature 
field of the plate with $N$ cracks and $2 n$ point heating sources is given by

$$
\begin{gathered}
F(z)=\sum_{k=1}^{n}\left[m_{a_{k}} \ln \left(z-a_{k}\right)+m_{b_{k}} \ln \left(z-b_{k}\right)\right] \\
T(x, y)=\sum_{k=1}^{n}\left\{m_{a_{k}} \ln \left[\left(x-a_{k}\right)^{2}+y^{2}\right]\right. \\
\left.+m_{b_{k}} \ln \left[\left(x-b_{k}\right)^{2}+y^{2}\right]\right\}
\end{gathered}
$$

\section{Temperature dependent thermal conductivity}

The thermal conductivity of metal may be regarded as the sum of lattice coefficient and electro conductivity. The power transmission of the lattice and free electrons is influenced by the temperature. This influences the thermal conductivity [7]. Suppose that the variation of thermal conductivity coefficient with the temperature is linear:

$\lambda=\lambda_{0}\left(1+\beta_{\lambda} T\right)$

The governing equation for the temperature then becomes [8]

$\frac{\partial}{\partial x}\left(\lambda \frac{\partial T}{\partial x}\right)+\frac{\partial}{\partial y}\left(\lambda \frac{\partial T}{\partial y}\right)=0$

Now define a new variable

$H=\int_{0}^{\mathrm{T}} \lambda \mathrm{d} T \quad$ with $\frac{\partial H}{\partial T}=\lambda$

Therefore, Eq. (25) can be transformed into the classical Laplace equation:

$\frac{\partial^{2} H}{\partial x^{2}}+\frac{\partial^{2} H}{\partial y^{2}}=0$

The procedure for of solving $H$ is well known. Hence, $m_{a_{k}}$ and $m_{b_{k}}$ in Eq. (14) follows from the relation $\partial H / \partial T=\lambda$. The temperature can then be obtained from Eq. (23).

\section{Stress field with collinear cracks}

Because the problem is symmetrical about the axis $o x$, it suffices to solve the semi-plane problem with mixed boundary conditions [9]. Now, also let $L=\sum_{k=1}^{n} L_{k}$ such that $L^{\prime}$ stands for the uncracked portion of the axis ox. Analysis will be on the lower semi-plane. The basic equations of this field are given by $[4,5,10]$

$$
\begin{aligned}
& \sigma_{y}+\sigma_{x}=2\lfloor\Phi(z)+\overline{\Phi(z)}\rfloor \\
& \sigma_{y}-\mathrm{i} \tau_{x y}=\Phi(z)-\Phi(\bar{z})+(z-\bar{z}) \overline{\Phi^{\prime}(z)} \\
& 2 \mu \frac{\partial}{\partial x}(u+\mathrm{i} v)=\kappa \Phi(z)+\Phi(\bar{z})-(z-\bar{z}) \overline{\Phi^{\prime}(z)}+\beta F(z)
\end{aligned}
$$

$X+\mathrm{i} Y=-\mathrm{i}[\varphi(z)-\varphi(\bar{z})+(z-\bar{z}) \overline{\Phi(z)}]_{a^{\prime}}^{b^{\prime}}$

where $\Phi(z)$ is the harmonic function of the field; $(u, v)$ are the displacement components. Note that $(X, Y)$ are components of the force vector acting on a curve $a^{\prime} b^{\prime} . \mu$ is the material's shear modulus with $\kappa=3-4 v$. Moreover, $\beta=2 E \alpha_{t}, v$ is the Poisson's ratio, $E$ is the elastic modulus and $\alpha_{t}$ is the coefficient of thermal expansion.

Because of the point heat sources at $a_{k}, b_{k}$ $(k=1,2, \ldots, n)$, the harmonic function $\Phi(z)$ near the crack tip $a_{k}$ and $b_{k}$ can be written as

$\Phi(z)=\sum_{k=1}^{n}\left[A_{a k}(z)+A_{b k}(z)\right]+\Phi_{*}(z)$,

when $z \rightarrow a_{k}$ or $z \rightarrow b_{k}$

$\Phi(z)=\sum_{k=1}^{n}\left[B_{a k}(z)+B_{b k}(z)\right]+\Phi_{*}(z)$,

when $z \rightarrow \overline{a_{k}}$ or $z \rightarrow \overline{b_{k}}$

where

$$
\begin{aligned}
& A_{a_{k}}(z)=\frac{\beta}{1+\kappa} m_{a_{k}} \ln \left(z-a_{k}\right), \\
& A_{b_{k}}(z)=\frac{\beta}{1+\kappa} m_{b_{k}} \ln \left(z-b_{k}\right) \quad(k=1,2, \ldots, n) \\
& B_{a_{k}}(z)=-\frac{\beta}{1+\kappa} m_{a_{k}} \ln \left(z-\bar{a}_{k}\right), \\
& B_{b_{k}}(z)=\frac{\beta}{1+\kappa} m_{b_{k}} \ln \left(z-\bar{b}_{k}\right) \quad(k=1,2, \ldots, n)
\end{aligned}
$$


Now, let

$A(z)=\sum_{k=1}^{n}\left[A_{a_{k}}(z)+A_{b_{k}}(z)\right]$
$B(z)=\sum_{k=1}^{n}\left[B_{a_{k}}(z)+B_{b_{k}}(z)\right]$

If the displacement boundary conditions are known on $L$, stress boundary conditions can be written as

$u+\mathrm{i} v=0 \quad\left(\right.$ on $\left.L^{\prime}\right), \quad \sigma_{y}-\mathrm{i} \tau_{x y}=0 \quad($ on $L)$

From Eqs. (31) and (32), the boundary condition of $\Phi(z)$ becomes

$\kappa \Phi^{-}(t)+\Phi^{+}(z)=g_{0}(t)\left(\right.$ on $\left.L^{\prime}\right)$

$\Phi^{-}(t)-\Phi^{+}(z)=0 \quad($ on $L)$

where

$g_{0}(t)=-\beta F(t)$

The values of $\Phi(z)$ at $a_{k}$ and $b_{k}$ are determined by Eq. (29). In this case, the solution of the boundary problem stated by Eq. (35) is given by

$\Phi(z)=A(z)+B(z)+\frac{\chi(z)}{2 \pi \mathrm{i}} \int_{L} \frac{f_{0}(\tau) \mathrm{d} \tau}{\chi^{+}(\tau)}+\chi(z) P_{n}(z)$

Note that

$$
\begin{aligned}
& P_{n}(z)=c_{0} z^{n}+c_{1} z^{n-1}+\cdots+c_{n} \\
& \chi(z)=\prod_{k=1}^{n}\left(z-a_{k}\right)^{-(1 / 2)}\left(z-b_{k}\right)^{-(1 / 2)}
\end{aligned}
$$

From Eqs. (28)-(30), the following can be obtained

$$
\begin{aligned}
& \sigma_{y}+\sigma_{x}=4 \operatorname{Re}[\Phi(z)]=-4 \beta \sum_{k=1}^{n}\left[m_{a_{k}} \ln \left|z-a_{k}\right|\right. \\
& \left.+m_{b_{k}} \ln \left|z-b_{k}\right|\right] \\
& \sigma_{y}-\mathrm{i} \tau_{x y}=-\beta \sum_{k=1}^{n}\left(m_{a_{k}} \ln \frac{z-a_{k}}{\bar{z}-a_{k}}+m_{b_{k}} \ln \frac{z-b_{k}}{\bar{z}-b_{k}}\right) \\
& +2 \beta y i \sum_{k=1}^{n}\left[\frac{m_{a_{k}}}{\bar{z}-a_{k}}+\frac{m_{b_{k}}}{\bar{z}-b_{k}}\right]
\end{aligned}
$$

The stresses $\sigma_{y}, \sigma_{x}$ and $\tau_{x y}$ can be obtained by separating the real and imaginary parts in the above equations. The modulus of elasticity $E$ and the coefficient of expansion for different temperatures can be found in handbooks.

\section{Strain energy density function}

While many fracture criteria are available, the strain energy density criterion will be used because of its generality for determining the crack path and the critical load to initiate crack growth. In this criterion, a strain energy density factor $S$ is defined from the strain energy density function $\mathrm{d} W / \mathrm{d} V$ using the relation $\mathrm{d} W / \mathrm{d} V=S / r$.

The distance $r$ is measured from the crack tip. Using the asymptotic crack tip stress field, $S$ becomes independent of $r$. It depends only on the angular distribution of the stress field. In general, $S$ can depend on $r$ for the relation $\mathrm{d} W / \mathrm{d} V=S / r$ hold in general as $S$ takes on the interpretation of the area under the $\mathrm{d} W / \mathrm{d} V$ versus distance $r$ curve. A finite distance $r$ is always kept between the crack tip and the element under consideration. The $S$ or $\mathrm{d} W / \mathrm{d} V$ criterion applies to all materials and external disturbances including thermal and moisture variations. The loading can in general be applied in an arbitrary direction such that the direction of crack initiation is not known but must be determined without additional assumptions.

Application of the criterion can be found in [3]. It requires a knowledge of the strain energy density function

$\frac{\mathrm{d} W}{\mathrm{~d} V}=\frac{1}{2 E}\left[(1+v) \sigma_{i j} \sigma_{i j}-v \sigma_{k k}\right]$

For linear and isotropic elasticity, $\mathrm{d} W / \mathrm{d} V$ can be separated into two parts. One refers to the distortional strain energy density given by

$$
\begin{aligned}
\left(\frac{\mathrm{d} W}{\mathrm{~d} V}\right)_{d}= & \frac{1+v}{6 E}\left[\left(\sigma_{1}-\sigma_{2}\right)^{2}+\left(\sigma_{2}-\sigma_{3}\right)^{2}\right. \\
& \left.+\left(\sigma_{3}-\sigma_{1}\right)^{2}\right]
\end{aligned}
$$

and the other to the dilatational the strain energy density expressed by

$$
\left(\frac{\mathrm{d} W}{\mathrm{~d} V}\right)_{V}=\frac{1-2 v}{6 E}\left(\sigma_{1}+\sigma_{2}+\sigma_{3}\right)^{2}
$$


where $\sigma_{j}(j=1,2,3)$ are the principal stresses. According to Eqs. (40) and (41), the strain energy density near crack tip when discharging can be obtained so that the path of crack propagation can be predicted by the strain energy density.

\section{Temperature and the stress field for two collinear cracks}

Consider two collinear cracks in a plate, Fig. 2. An electric current with intensity $J_{0}$ is activated at remote distance from the cracks. Here,

$A_{1}(-3 a, 0), B_{1}(-a, 0), A_{2}(a, 0), B_{2}(3 a, 0)$

and $L=L_{1} \cup L_{2}$

Note that $L^{\prime}$ is the uncracked ligaments (except $L$ ) along the axis $o x$. From Eqs. (7) and (11), the following is obtained

$$
\Phi^{\mathrm{T}}(z)=\frac{J_{0}}{\mathrm{i} \sqrt{\left(z^{2}-a^{2}\right)\left(z^{2}-9 a^{2}\right)}}\left[z^{2}-9 a^{2} \frac{E(k)}{K(k)}\right]
$$

where $E(k), K(k)$ are respectively the first and second elliptic integrals with argument $k$ where $k^{2}=1-(1 / 3)^{2}$.

From Eqs. (23) or (25), $T(x, y)$ may be written as

$$
\begin{aligned}
T(x, y)= & \sum_{k=1}^{2}\left\{m_{a_{k}} \ln \left[\left(x-a_{k}\right)^{2}+y^{2}\right]\right. \\
& \left.+m_{b_{k}} \ln \left[\left(x-b_{k}\right)^{2}+y^{2}\right]\right\}
\end{aligned}
$$
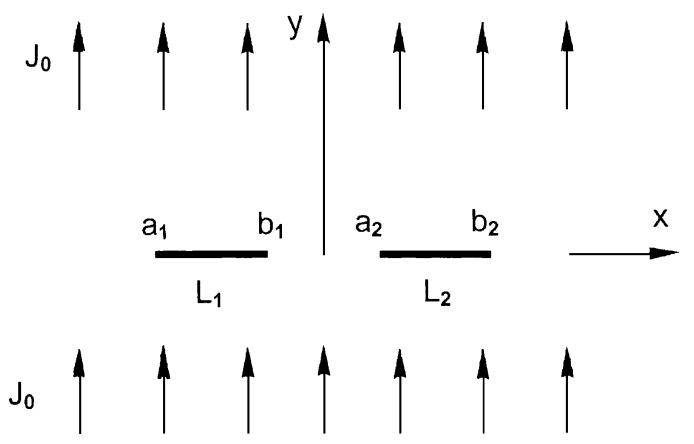

Fig. 2. A thin current carrying plate with two collinear cracks.
It should be noted that

$a_{1}=-3 a, \quad b_{1}=-a, \quad a_{2}=a, \quad b_{2}=3 a$

and $A_{a_{k}}, A_{b_{k}}$ may be found by Eq. (17). From Eqs. (40) and (41), the following is known

$$
\begin{aligned}
\sigma_{y}+\sigma_{x}= & 4 \operatorname{Re}[\Phi(z)] \\
= & 4 \beta \sum_{k=1}^{2}\left[m_{a_{k}} \ln \left(z-a_{k}\right)+m_{b_{k}} \ln \left(z-b_{k}\right)\right] \\
\sigma_{y}-\mathrm{i} \tau_{x y}= & \beta \sum_{k=1}^{2}\left(m_{a_{k}} \ln \frac{z-a_{k}}{\bar{z}-a_{k}}+m_{b_{k}} \ln \frac{z-b_{k}}{\bar{z}-b_{k}}\right) \\
& +2 \beta y \mathrm{i} \sum_{k=1}^{2}\left[\frac{m_{a_{k}}}{\bar{z}-a_{k}}+\frac{m_{b_{k}}}{\bar{z}-b_{k}}\right]
\end{aligned}
$$

\section{Example}

A thin plate with two collinear cracks in Fig. 2 is made of high temperature resistance alloy GH2132. The plate thickness is $2 h=2 \mathrm{~mm}$ and $a=10 \mathrm{~mm}$. The coefficient of heat conduction is assumed to have a linear variation with time so that $\beta_{T}$ can be found from its values at 100 and 900 ${ }^{\circ} \mathrm{C}$. This gives $\beta_{T}=1.048 \times 10^{3}$. The electrical conductivity of the alloy is $\sigma=1.12 \times 10^{6}(\Omega \mathrm{m})^{-1}$. The linear expansion coefficient and the elastic modulus of the material are given in Tables 1 and 2 [11]. In Fig. 3, curves a and c show the temperature near the crack tip $a_{1}$ and $b_{1}$ when the variation of the heat conduction coefficient is not considered. Curves $\mathrm{b}$ and $\mathrm{d}$ show the temperature when the variation of the heat conduction coefficient is considered. Temperatures at the crack tip $a_{2}$ and $b_{2}$ are the same as those at $a_{1}$ and $b_{1}$.

In Fig. 4, the curves 1 and 2 describe the value of $\sigma_{x}$ at $1 \mathrm{~mm}$ from the crack tip. The current intensity is changed. In Fig. 5, when the crack size is changed, the curves 1 and 2 describe the value of $\sigma_{x}$ at $1 \mathrm{~mm}$ from the crack tip. The current intensity is $J_{0}=1.3 \times 10^{8} \mathrm{~A} / \mathrm{m}^{2}$ which shows the values of $\sigma_{x}$ as a function of distance along the line collinear with the crack. Displayed in Fig. 6 is the distribution of strain energy density at crack tip $b_{2}$. The crack propagation path is shown when the 
Table 1

Coefficients of linear expansion of alloy at different temperature

\begin{tabular}{ll}
\hline$T\left({ }^{\circ} \mathrm{C}\right)$ & $\alpha_{T}$ \\
\hline 100 & 15.37 \\
200 & 16.09 \\
300 & 16.31 \\
400 & 16.84 \\
500 & 17.58 \\
600 & 18.06 \\
700 & 18.74 \\
800 & 19.62 \\
900 & 20.45 \\
\hline
\end{tabular}

Here the values of $\alpha_{t}$ are $\alpha_{T} \times 10^{-6} \mathrm{C}^{-1}$.

Table 2

Elastic moduli of alloy at different temperature

\begin{tabular}{ll}
\hline$T\left({ }^{\circ} \mathrm{C}\right)$ & $E(\mathrm{GPa})$ \\
\hline 20 & 198 \\
100 & 193 \\
200 & 186 \\
300 & 181 \\
400 & 173 \\
500 & 165 \\
600 & 157 \\
700 & 150 \\
800 & 139 \\
\hline
\end{tabular}

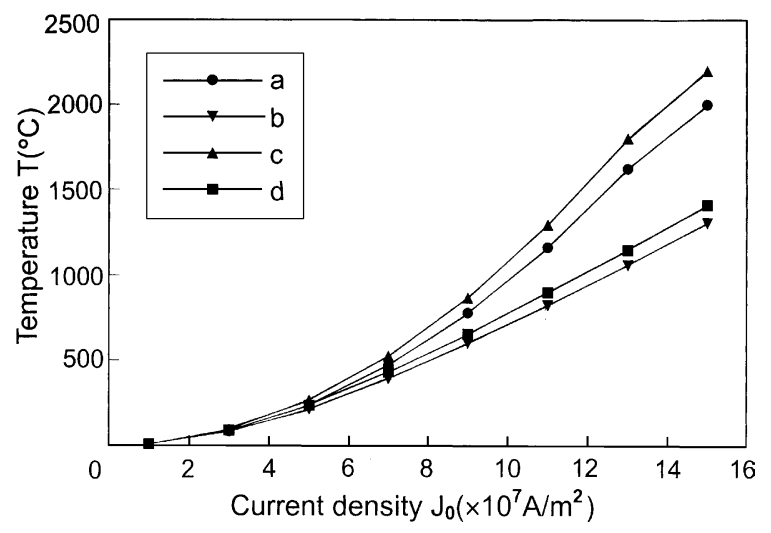

Fig. 3. Curve $T \sim J_{0}$.

discharging current density is $J_{0}=1.3 \times 10^{8} \mathrm{~A} / \mathrm{m}^{2}$. Fig. 7 shows the distribution of the strain energy density for different current density at crack tip $b_{2}$. In Fig. 8, the variation of the ratio of dilatational

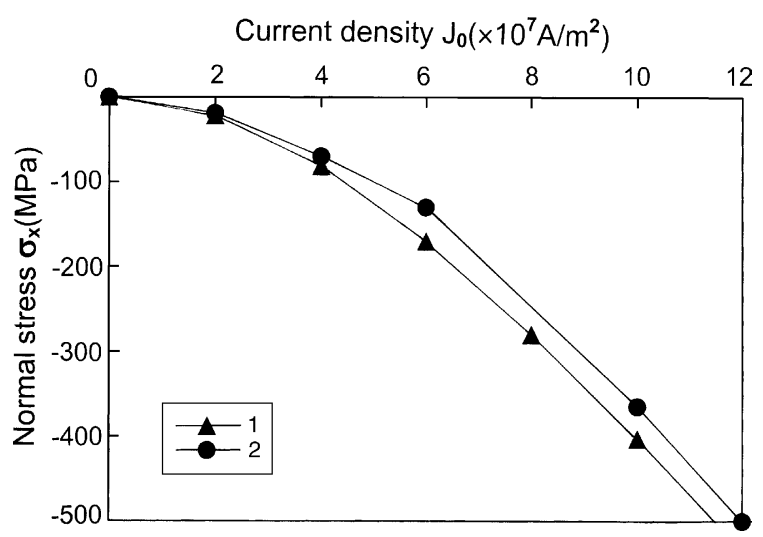

Fig. 4. Curve $\sigma_{x} \sim J_{0}$.

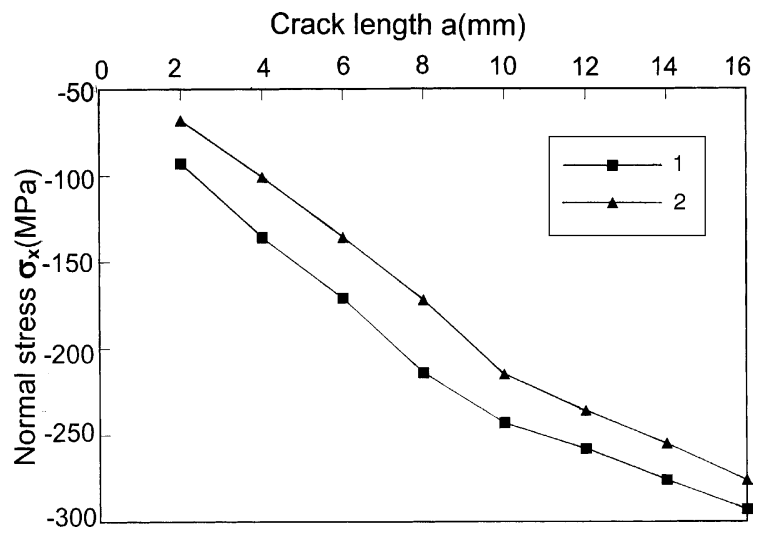

Fig. 5. Curve $\sigma_{x} \sim a$.

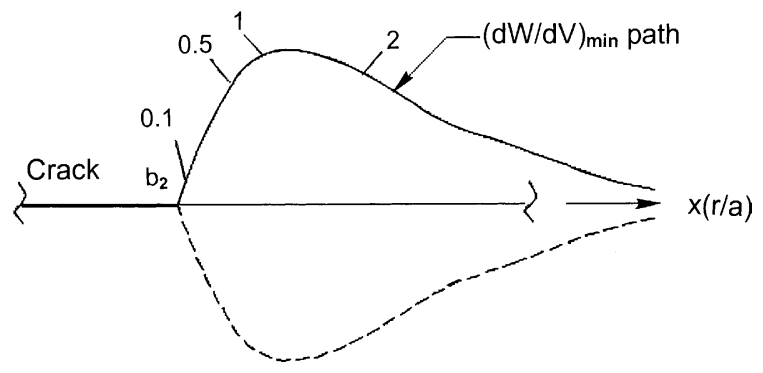

Fig. 6. Fracture trajectory.

and distortional energy density factor with angel $\theta$ is shown. Here, $r$ is the distance measured from the crack tip $b_{2}$ while $\theta$ is the angle between $r$ and the $x$-axis. 


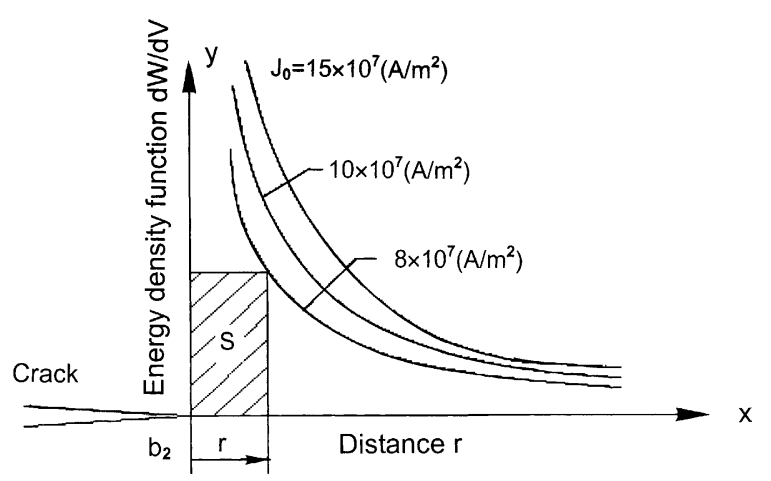

Fig. 7. The relations of strain energy density with $J_{0}$.

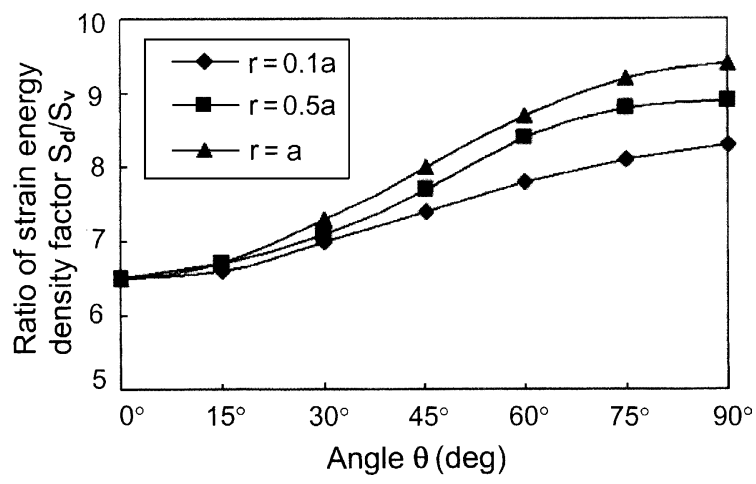

Fig. 8. The variation of ratio of dilatational and distortional energy with angel $\theta$.

\section{Conclusions}

From the results obtained in this work, the following conclusions can be made:

- Fig. 3 shows that the influence of temperature on the variation of the coefficient of heat conduction cannot be ignored when the temperature is high. The influence of the current intensity on the temperature is very pronounced. Crack arrest behavior depends sensitively on these factors.

- As shown in Fig. 4, the stress near the crack tip is compressive. The greater the current intensity, the larger is the stress magnitude.

- From Fig. 5, it can be observed that when the variation of the size of $a$ is small, the stress variation is very large. When the size of $a$ is more than, say $13 \mathrm{~mm}$, changes in the size of $a$ have a smaller influence on the stress.

- Fig. 7 shows that the strain energy density will increase when the discharging current density increases. It is also shown that the strain energy density intensity tends to increase as the crack tip is approached. Fig. 8 shows that the distortional energy is much bigger than the dilatational energy near the crack tip. However, they do not occur at the same location. The former is away from the line of prospective crack growth. What should be kept in mind is the order of events implicit in the equilibrium theory of mechanics. That is distortion will always take place over a longer time period prior to dilatation. The latter takes place over a much shorter time interval and more abruptly. The equilibrium nature of mechanics only presents the space and time average results. This prevents a detail account of the actual physical nature of the phenomenon of yield and fracture. Refer to [3] for a detail description of the situation near the crack tip.

\section{Acknowledgements}

The project is supported by the National Natural Science Foundation of China (50275128) and National Laboratory of Non-linear Mechanics in Institute of Mechanics of The Chinese Academy of Sciences.

\section{References}

[1] B. Dexian, Theory of Electro-magnetic Field, Press of the Electric Industry, Beijing, 1985, pp. 207-232.

[2] G.C. Sih, Thermal behavior of an infinite medium with collinear cracks, Applied Mechanics, vol. C, Heat Transfer, USSR, 1965, 87, No.2, P157-162 (in Russian).

[3] G.C. Sih, Mechanics of Fracture Initiation and Propagation, Kluwer Academic Publishers, Boson, 1991, pp. 182212.

[4] N.I. Mushelishvili, Translated by Huiyuan Zhao. Some Basic Problem of the Mathematical Theory of Elasticity, Press of Science, Beijing, 1958, pp. 196-407.

[5] B.A. Kudriavcev, V.Z. Parton, Thermal magneto elasticity field of a body with a semi-infinite crack, Applied Math and Mechanics 44 (5) (1950) (in Russian). 
[6] G.S. Kit, M.G. Krivcun, Two-Dimensional Problem of Thermal Elasticity in A Solid with Cracks, Jifu, Press of Scientific Thinking, 1983, pp. 18-40 (in Russian).

[7] E.R.G.R.M. Derik, Translated by Hangqing, Analyzing Heat Transfer and Mass Transfer, Press of Science, Beijing, 1983, pp. 31-68.

[8] W. Buxian, Engineering Theory of Heat Transfer and Mass Transfer, Press of Science, Beijing, 1998, pp. 98139.
[9] N.-A. Mahmoud, N. Naotake, Study of edge crack problem in a semi-infinite functionally graded medium with two dimensionally nonhomogeneous coefficients of thermal expansion under thermal load, Journal of Thermal Stress 19 (1996) 863-888.

[10] I.A. Prusov, Some Problem of Thermal Elasticity, Min-sike, Press of National University of White Russia, 1972, pp. 12-40.

[11] Practical Handbook of Engineering Materials, Standard Press of China, Beijing, 1989, pp. 157-178. 